\title{
openheart Effectiveness of an impedance cardiography guided treatment strategy to improve blood pressure control in a real-world setting: results from a pragmatic clinical trial
}

\author{
Luyan Wang, ${ }^{1}$ Yuan Lu (D),${ }^{2,3}$ Hongyi Wang, ${ }^{1}$ Jianlei Gu, ${ }^{4,5}$ Zheng J Ma, ${ }^{4,6}$ \\ Zheng Lian, ${ }^{6}$ Zhiying Zhang, ${ }^{7}$ Harlan Krumholz, ${ }^{3,8}$ Ningling Sun ${ }^{1}$
}

\begin{abstract}
- Additional supplemental material is published online only. To view, please visit the journal online (http://dx.doi.org/10. 1136/openhrt-2021-001719).

To cite: Wang L, Lu Y, Wang $\mathrm{H}$, et al. Effectiveness of an impedance cardiography guided treatment strategy to improve blood pressure control in a real-world setting: results from a pragmatic clinical trial. Open Heart 2021;8:e001719.

doi:10.1136/

openhrt-2021-001719
\end{abstract}

$\mathrm{YL}$ and $\mathrm{LW}$ are joint first authors

Received 12 May 2021

Accepted 2 August 2021
D Check for updates

(c) Author(s) (or their employer(s)) 2021. Re-use permitted under CC BY. Published by BMJ.

For numbered affiliations see end of article.

Correspondence to

Dr Ningling Sun; sunnl@263.net

\section{ABSTRACT}

Objective To test the effectiveness of an impedance cardiography (ICG) guided treatment strategy on improving blood pressure (BP) control in real-world clinical practice.

Design A single-centre, pragmatic randomised trial. Setting A hypertension clinic of the Peking University People's Hospital in Beijing, China.

Participants Adults who sought outpatient care for hypertension in the hypertension clinic at the Peking University People's Hospital between June and December 2019.

Interventions A computerised clinical decision support of recommending treatment choices to providers based on patients' haemodynamic profiles measured by ICG.

Main outcome measures Changes in systolic BP (SBP) and diastolic BP (DBP) levels at the follow-up visit 4-12 weeks after baseline. Secondary outcomes included achievement of BP goal of $<140 / 90 \mathrm{~mm} \mathrm{Hg}$ and the changes in $\mathrm{BP}$ by baseline $\mathrm{BP}$, age, sex and body mass index (BMI).

Results A total of 102 adults (mean age was $54 \pm 14$ years; $41 \%$ were women) completed the study. The mean baseline SBP was 150.9 (SD of 11.5) $\mathrm{mm} \mathrm{Hg}$ and mean baseline DBP was 91.1 (11.3) $\mathrm{mm} \mathrm{Hg}$. At the follow-up visit, the mean SBP and DBP decreased by 19.9 and $11.3 \mathrm{~mm} \mathrm{Hg}$ in the haemodynamic group, as compared with 12.0 and $4.9 \mathrm{~mm} \mathrm{Hg}$ in the standard care group ( $p$ value for difference between groups $<0.001$ ). The proportion of patients achieving BP goal of $<140 / 90 \mathrm{~mm}$ $\mathrm{Hg}$ in the haemodynamic group was $67 \%$, as compared with $41 \%$ in the standard care group $(p=0.017)$. The haemodynamic group had a larger effect on BP reduction consistently across subgroups by age, sex, BMI and baseline BP.

Conclusions An ICG-guided treatment strategy led to greater reductions in BP levels than were observed with standard care in a real-world population of outpatients with hypertension. There is a need for further validation of this strategy for improving blood pressure treatment selection.

Trial registration number NCT04715698.

\section{Key questions}

What is already known about this subject?

- Hypertension is a hemodynamic-related disorder characterized by abnormalities of the cardiac output, systemic vascular resistance, or a combination of both. Measurement of the various hemodynamic parameters using impedance cardiography (ICG) in stable patients with hypertension provides information that may enable more effective targeted drug management.

What does this study add?

- This study shows an ICG-guided treatment strategy could lead to greater reductions in blood pressure levels than were observed with standard care in a real-world population of outpatients with hypertension.

How might this impact on clinical practice?

- As clinical care is moving towards precision medicine, our findings identify the needs of more refined hemodynamic measurement to facilitate personalized treatment in patients with hypertension.

\section{BACKGROUND}

Hypertension is a haemodynamic-related disorder characterised by abnormalities of the cardiac output $(\mathrm{CO})$, systemic vascular resistance (SVR) or a combination of both. ${ }^{1}$ Despite that hypertension is routinely diagnosed and managed based on degree of blood pressure (BP) elevation alone, patients with similar degree of $\mathrm{BP}$ elevation can have different underlying haemodynamic profiles. ${ }^{23}$ These variations in haemodynamic profiles may have important implications for treatment selection because the choice for patients with a higher $\mathrm{CO}$ might be different than for those with a higher SVR. Selecting treatment strategies based on haemodynamic 
profiles for patients with hypertension may improve BP control.

Impedance cardiography (ICG) is a safe and accurate non-invasive tool to measure haemodynamic parameters ${ }^{45}$ that can be performed in the outpatient setting. ${ }^{67}$ Measurement of the various haemodynamic components using ICG in stable patients with hypertension provides information that may enable more effective targeted drug management. Although several previous studies have used ICG to evaluate haemodynamic parameters and demonstrated that ICG-guided therapy improves BP control, ${ }^{7-9}$ they used a traditional randomised controlled trial design, in which the operationalisation of the intervention had stricter instructions and patients were more frequently monitored than routine clinical care. Whether an ICG-guided strategy for hypertension treatment can lead to improvements in BP control in real-world clinical settings has been rarely tested. Additionally, previous studies were all conducted in the $\mathrm{USA}^{89}$; no study has focused on low-income and middle-income counties where healthcare resources are limited, patient characteristics and clinical practice patterns are different.

Accordingly, we conducted a pragmatic randomised trial to produce preliminary data about the effectiveness of ICG-guided strategies for patients with hypertension in routine clinical care in China. We hypothesised that selecting antihypertensive therapy based on each patient's haemodynamic profile measured by ICG could lead to more effective BP reduction and hypertension control than standard care in hypertensive patients in a real-world setting.

\section{METHODS \\ Eligibility}

The study population was patients who sought outpatient care for hypertension in the hypertension clinic of the Cardiology Department at the Peking University People's Hospital between June and December 2019 in Beijing, China. Patients were eligible if they were 18-85 years old, were local residents, had a diagnosis of essential hypertension and were currently on less than four antihypertensive medications of different classes with systolic BP (SBP) of $\geq 140 \mathrm{~mm} \mathrm{Hg}$ or diastolic BP (DBP) of $\geq 90 \mathrm{~mm}$ $\mathrm{Hg}$. If patients were on a combination antihypertensive drug, they would be considered on multiple classes of antihypertensive drugs. Patients were excluded if they were already on four or more antihypertensive agents of different classes (considered as resistant hypertension); had on-site SBP of $<140 \mathrm{~mm} \mathrm{Hg}$ and DBP of $<90 \mathrm{~mm} \mathrm{Hg}$; had secondary hypertension, severe renal disease, cancer, severe valvular disease, cerebrovascular event within 6 months, atrial fibrillation; or had uncontrolled diabetes with fasting blood glucose of $11.1 \mathrm{mmol} / \mathrm{L}$.

\section{Randomisation and procedure}

After informed consent, patients meeting inclusion/ exclusion criteria were randomised in a 1:1 ratio to the haemodynamic group or the standard care group. Simple randomisation was performed using a random number generator with concealed allocation. Randomisation was performed at the patient rather than the provider level, as outpatients at the participating clinic may be cared for by different providers throughout the study. All study investigators were blinded to patient randomisation status until enrolment was complete.

Patients' information including age, sex, weight, height, BP and antihypertensive medications was collected by nurses during the outpatient visit. Weight was measured to the nearest $0.1 \mathrm{~kg}$ with patients wearing light indoor clothing and no shoes. Height was measured to the nearest $0.1 \mathrm{~cm}$, using a portable stadiometer (Omron HNJ-318; Omron Corporation, Kyoto, Japan) with patients standing without shoes and heels against the wall. BP was measured on the right upper arm after $5 \mathrm{~min}$ of rest in a seated position using an electronic BP monitor (Omron HBP-9020; Omron Corporation). ICG data were collected by trained technicians at each visit in all patients, but ICG findings were not revealed in the standard arm to physicians or patients. ICG was performed with patients in the supine position, resting for 3 min before measurement. By applying a constant, low amplitude, high-frequency, alternating electrical current to the thorax, ICG device measures the corresponding voltage to detect beat-to-beat changes in thoracic electrical resistance, known as impedance and with it stroke volume is estimated. ${ }^{10} 11$ Then, using heart rate, mean arterial $\mathrm{BP}$ and $\mathrm{BMI}$, other haemodynamic parameters are calculated, including $\mathrm{CO}$, cardiac index (CI), SVR, SVR index (SVRI), arterial stiffness index (AS) and a volume parameter - thoracic blood saturation ratio (TBR). ${ }^{12}$ The ICG device used (CHM P2505, designed by Beijing Li-Heng Medical Technologies, manufactured by Shandong Baolihao Medical Appliances) was developed based on improved hardware and advanced digital filtering algorithms ${ }^{13}$ and has been validated versus both invasive thermodilution and non-invasive echocardiography in different settings. ${ }^{14-16}$

\section{Intervention}

After randomisation, therapy was initiated in all patients. Physicians in both groups were encouraged to prescribe medications consistent with the 2018 Chinese hypertension guideline, ${ }^{17}$ their clinical judgement, and patient clinical characteristics. In the haemodynamic group, physicians were provided with patients' ICG findings and a computerised clinical decision support of recommended treatment choices based on patients' haemodynamic profiles. Specifically, the clinical decision support system determined the haemodynamic phenotype of a patient in three steps: first, the computer system calculated the population mean and SD of each haemodynamic parameter (eg, HR, CI, AS, SVRI, TBR) given patient's gender, age, weight, height and BMI, using data from a large sample of 114198 generally healthy Chinese adults (see detailed description in online supplemental 


\section{Hypertension Hemodynamic Phenotyping (HDpro)} \& Antihypertensives Recommendations

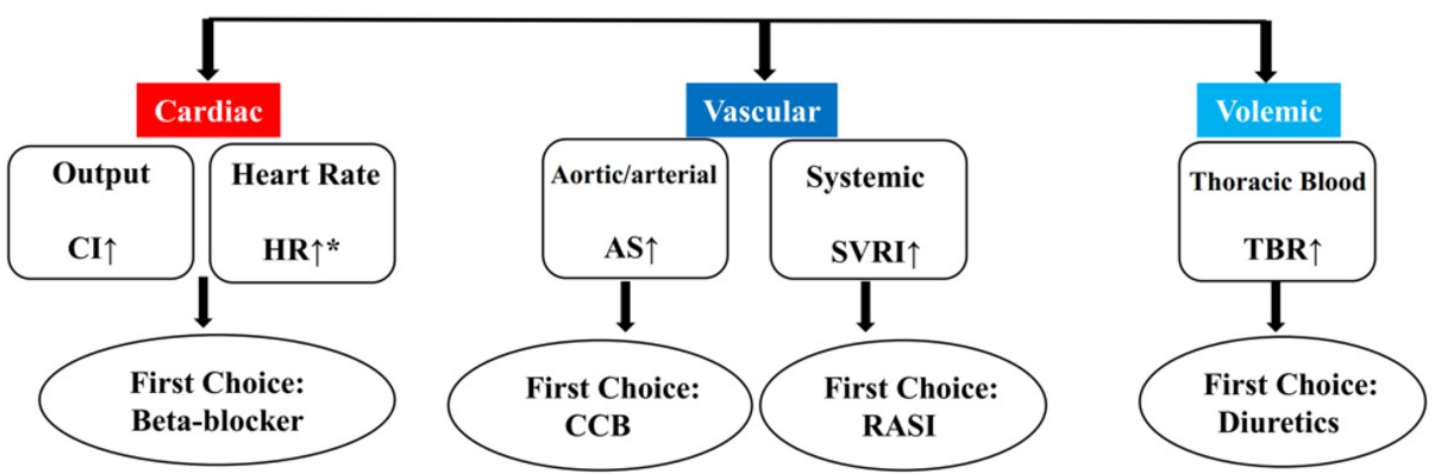

Figure 1 Suggested treatment strategy for the haemodynamic group. CCB, calcium channel blockers; RASI, reninangiotensin system inhibitors. In case of evaluation of multiple indicators, first consider the highest one, or considering drug combinations. In case that the recommended drug is already used, consider dose titration, switching to extended release formulation or switching to a different in the same drug class. Based on hemodynamic phenotyping, final drug choices should take in consideration comorbidity and other clinical info. $\uparrow:$ Value greater than "baseline + 1SD", baseline and SD based on large sample Chinese general population, adjusted for individual factors such as: age, sex, height and weight. HR $\uparrow *$ : HR>75 b/ min; But in case of low cardiac output (Cl<baseline-2SD), use beta-blockers cautiously. AS, aortic/arterial stiffness; $\mathrm{Cl}$, cardiac index; SVRI, systemic vascular resistance index; TBR, thoracic blood standing/supine ratio.

file 1$).{ }^{2}{ }^{3}$ Because haemodynamic parameters vary by age, gender, height and weight, we used personalised cutoffs as opposed to one-size-fits-all cutoffs to define haemodynamic phenotypes. Second, the computer system determined if the patient had an elevated haemodynamic parameter based on whether the patient's value was greater than the population mean plus one SD of the respective parameter. Finally, the clinical decision support categorised patients into four clinically relevant haemodynamic phenotypes, including cardiac phenotype (high HR or high CI), arterial vascular phenotype (high AS), peripheral vascular phenotype (high SVRI) and volemic phenotype (high TBR) ${ }^{18} 19$ These four haemodynamic phenotypes included cardiac phenotype (high HR or high CI), arterial vascular phenotype (high AS), peripheral vascular phenotype (high SVRI) and volemic phenotype (high TBR). Suggested treatment strategies were then provided for each phenotype (see details in figure 1). Physicians were instructed to use this information to guide decisions about pharmacological agents and dosing. Physicians could share ICG information with patients in the haemodynamic arm. In the standard care group, physicians were not provided with patients' ICG findings and were instructed to use their own clinical judgement to make treatment decisions. To minimise the potential confounding due to lifestyle modification, physicians in both groups were instructed not to prescribe non-pharmacological interventions as part of their treatment plans. All patients in both groups received education on the importance of medication compliance.

\section{Outcome measures}

All patients were required to return to the clinic for a follow-up visit between 4 and 12 weeks after the baseline visit. During the follow-up visit, BP was measured on the right upper arm after $5 \mathrm{~min}$ of rest in a seated position using an electronic BP monitor (Omron HBP9020). The technicians who measured BP were blinded to the intervention arm. The primary study end points were changes in SBP and DBP from baseline. Secondary study end points included (1) achievement of BP goal of $<140 / 90 \mathrm{~mm} \mathrm{Hg}$ and (2) changes in SBP and DBP by baseline BP, age, sex and BMI.

\section{Statistical analysis}

We described continuous variables as mean \pm SD and categorical variables as $\mathrm{n}(\%)$. Differences in continuous variables between treatment groups were examined by the Student's t-test and in categorial variables using Fisher's exact tests. Subgroup analysis was performed by baseline $\mathrm{BP}$, age, sex, BMI and haemodynamic phenotype. We used Breslow-Day test to test the consistency of different stratified OR across subgroups and used Forest plots for visualisation. We performed additional evaluation of changes in haemodynamic parameters between baseline and follow-up visit by pair-sample t-test. Statistical significance was defined as a two-tailed $\mathrm{p}<0.05$. All statistical analyses were conducted using R, V.3.4.1 (The R Foundation for Statistical Computing, Vienna, Austria). The study followed the guidelines for randomised trial, described in the Consolidated Standards of Reporting Trials statement. ${ }^{20}$ 
Table 1 Characteristics of study participants at baseline

\begin{tabular}{|c|c|c|c|}
\hline & $\begin{array}{l}\text { Overall } \\
(n=102)\end{array}$ & $\begin{array}{l}\text { Intervention } \\
\text { group } \\
(n=51)\end{array}$ & $\begin{array}{l}\text { Control } \\
\text { group } \\
(n=51)\end{array}$ \\
\hline Male, n (\%) & $60(59 \%)$ & $32(63 \%)$ & $28(55 \%)$ \\
\hline Female, n (\%) & $42(41 \%)$ & $19(37 \%)$ & $23(45 \%)$ \\
\hline Age, mean (SD) & $54 \pm 14.0$ & $55 \pm 12.4$ & $54 \pm 15.5$ \\
\hline $\mathrm{BMI}\left(\mathrm{kg} / \mathrm{m}^{2}\right)$ & $26.7 \pm 3.8$ & $26.4 \pm 3.7$ & $27.0 \pm 3.9$ \\
\hline $\mathrm{SBP}(\mathrm{mm} \mathrm{Hg})$ & $150.9 \pm 11.5$ & $151.8 \pm 12.6$ & $150.0 \pm 10.3$ \\
\hline $\mathrm{DBP}(\mathrm{mm} \mathrm{Hg})$ & $91.1 \pm 11.3$ & $92.7 \pm 9.6$ & $89.5 \pm 12.6$ \\
\hline HR (BPM) & $72 \pm 10.6$ & $73 \pm 11.2$ & $70.0 \pm 10.0$ \\
\hline $\mathrm{Cl}\left(\mathrm{L} / \mathrm{min} / \mathrm{m}^{2}\right)$ & $3.1 \pm 0.72$ & $3.1 \pm 0.64$ & $3.0 \pm 0.80$ \\
\hline AS (mm Hg/mL/b) & $0.82 \pm 0.33$ & $0.82 \pm 0.36$ & $0.82 \pm 0.30$ \\
\hline SVRI (dyn s m²/cm ${ }^{5}$ ) & $3017 \pm 731$ & $3057 \pm 678$ & $2975 \pm 720$ \\
\hline TBR (\%) & $0.78 \pm 0.11$ & $0.79 \pm 0.11$ & $0.76 \pm 0.10$ \\
\hline Diabetes, n (\%) & $25(25 \%)$ & $13(25 \%)$ & $12(24 \%)$ \\
\hline CHD, n (\%) & $4(4 \%)$ & $2(4 \%)$ & $2(4 \%)$ \\
\hline Stoke, n (\%) & $1(1 \%)$ & $1(2 \%)$ & $0(0 \%)$ \\
\hline CKD, n (\%) & $9(9 \%)$ & $7(14 \%)$ & $2(4 \%)$ \\
\hline
\end{tabular}

The data of the two groups were not statistically significant ( $p>0.05$ for all).

$\mathrm{AS}$, aortic resistance index; $\mathrm{BMI}$, body mass index; $\mathrm{CHD}$, coronary heart disease; $\mathrm{Cl}$, cardiac output index; CKD, chronic kidney disease; DBP, diastolic blood pressure; HR, heart rate; SBP, systolic blood pressure; SVRI, systemic vascular resistance index; TBR, thoracic blood volume saturation.

\section{Patient involvement}

No patients were involved in the development of the research question or the outcome measures, or in developing plans for the design and implementation of the study. The data are deidentified and, therefore, cannot be shared with the study participants directly.

\section{RESULTS}

\section{Characteristics of study population}

Between 1 June and 31 December 2019, we screened 201 patients presenting to the hypertension clinic for outpatient care, from which we excluded 87 individuals whose baseline BP value is less than $140 / 90 \mathrm{~mm} \mathrm{Hg}$, leaving 114 patients randomised to the intervention and control arms. We further excluded 12 patients who did not make follow-up visits within 4-12 weeks. Finally, a total of 102 patients (51 in the standard care group and 51 in the haemodynamic group) completed the study and were analysed (online supplemental figure 1). Among 102 patients, the mean age of $54 \pm 14$ years and $41 \%$ were female. Patients had a mean SBP of $150.9( \pm 11.5) \mathrm{mm} \mathrm{Hg}$, mean DBP of $91.1( \pm 11.3) \mathrm{mm} \mathrm{Hg}$, mean CI of $3.1( \pm 0.7)$ $\mathrm{L} / \mathrm{min} / \mathrm{m}^{2}$, mean SVRI of $3017( \pm 731)$ dynes $\mathrm{s} / \mathrm{cm}^{5} / \mathrm{m}^{2}$, mean heart rate of $72( \pm 10.6)$ beats/min (table 1$)$.

In the haemodynamic group, 13 patients had cardiac phenotype (high HR or high CI), 11 had arterial vascular phenotype (high AS), 30 had peripheral vascular
BP Reduction at Follow-up Visit

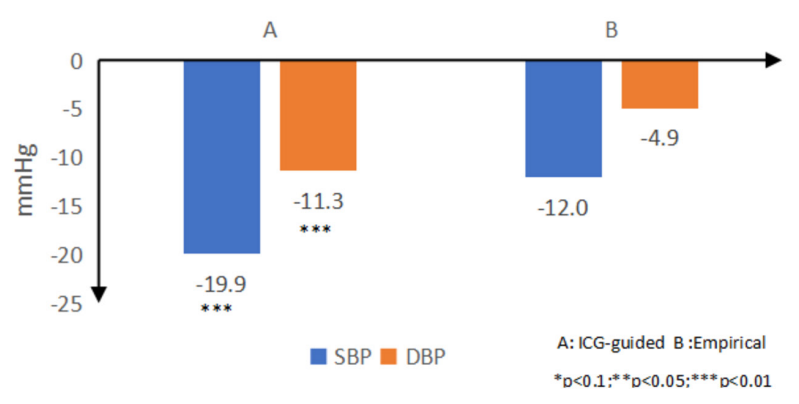

BP Control Rate at Follow-up Visit (Proportion $<140 / 90 \mathrm{mmHg}$ )

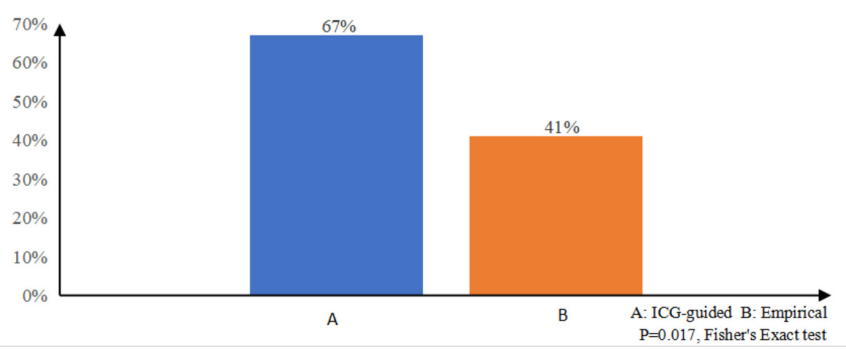

Figure 2 Blood pressure (BP) reduction and achievement of BP goals in haemodynamic and standard care groups. A: Haemodynamic group, B: standard care group. DBP, diastolic BP; ICG, impedance cardiography; SBP, systolic BP.

phenotype (high SVRI) and 17 volemic phenotype (high TBR), respectively. In the control group, 13 patients had cardiac phenotype, 18 had arterial vascular phenotype, 26 had peripheral vascular phenotype and 11 volemic phenotype, respectively. There were no statistically significant differences in the number and class of antihypertensive medications, patient demographic, clinical, BP or ICG variables at baseline between the haemodynamic group and the control group (table 1 and online supplemental tables 1-3). Baseline ICG variables and patient characteristics by haemodynamic phenotype were presented in online supplemental tables 1 and 2.

\section{Effect of the ICG-guided treatment strategy on BP control}

BP and ICG values at the baseline and follow-up visit as well as their differences between the two visits are shown in online supplemental table 4 and figure 2. Both SBP and DBP reductions were significantly greater in the haemodynamic group from baseline to follow-up visit compared with the standard care group (SBP reductions: $19.9 \pm 10.7$ vs $12.0 \pm 11.8 \mathrm{~mm} \mathrm{Hg}, \mathrm{p}<0.001$; DBP reduction: $11.3 \pm 6.2$ vs $4.9 \pm 9.9 \mathrm{~mm} \mathrm{Hg}, \mathrm{p}<0.001)$. Final BP was lower in the haemodynamic group compared with the standard care group (SBP: $131.9 \pm 10.9$ vs $138.0 \pm 13.7 \mathrm{~mm} \mathrm{Hg}, \mathrm{p}<0.001$; DBP: $81.4 \pm 7.7$ vs $84.6 \pm 12.9 \mathrm{~mm} \mathrm{Hg}, \mathrm{p}<0.001)$. The proportion of patients achieving BP goal of $<140 / 90 \mathrm{~mm} \mathrm{Hg}$ was also larger in the haemodynamic group compared with the standard care group ( $67 \%$ vs $41 \%$; $\mathrm{p}=0.017$ ).

Subgroup analyses by patient gender (men vs women), age ( $\geq 50$ years vs $<50$ years), BMI $(\geq 24 \mathrm{vs}<24 \mathrm{~kg} / \mathrm{m} 2)$ and baseline BP level (baseline SBP $\geq 160$ vs $140-159 \mathrm{~mm}$ 


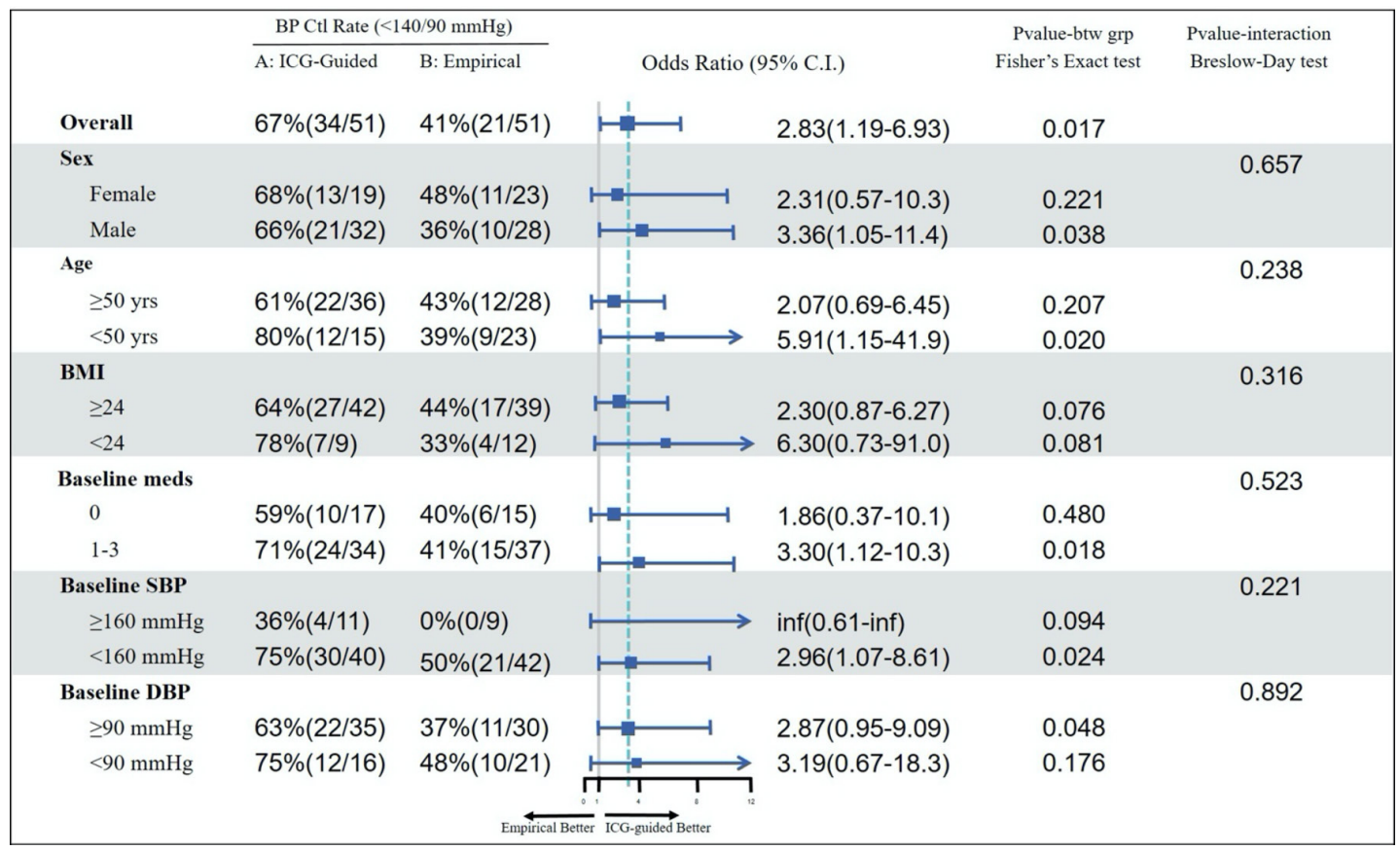

Figure 3 Achievement of BP goals by age, sex, BMI, baseline BP, use of medication at baseline. AS, aortic resistance index; BMI, body mass index; BP, blood pressure; DBP, diastolic BP; ICG, impedance cardiography; SBP, systolic BP; SVRI, systemic vascular resistance index.

$\mathrm{Hg}$; baseline DBP $\geq 90 \mathrm{vs}<90 \mathrm{~mm} \mathrm{Hg}$ ) have consistently shown a greater $\mathrm{BP}$ reduction in the haemodynamic group compared with the standard care group. The differences between the two groups were statistically significant for all subgroups, except for DBP in men, SBP in the age of $<50$ years and DBP in BMI of $<24 \mathrm{~kg} /$ $\mathrm{m}^{2}$ where the differences between the two groups were non-significant. The proportion of patients achieving BP goal of $<140 / 90 \mathrm{~mm} \mathrm{Hg}$ was statistically significantly larger in the haemodynamic group compared with the standard care group for subgroups of men, age of $<50$ years, baseline SBP of $<160 \mathrm{~mm} \mathrm{Hg}$ and baseline DBP of $\geq 90 \mathrm{~mm} \mathrm{Hg}$ (figure 3 ).

Figure 4 showed BP reduction between two treatment groups by haemodynamic phenotypes. BP reduction was significantly larger in haemodynamic group compared with the standard care group for patients with hyperdynamic phenotype (high HR or high CI), arterial hyperresistive phenotype (high AS) and peripheral artery hyper-resistive phenotype (high SVRI). BP reduction was not statistically significant in patients with high volume phenotype (high TBR).

\section{BP Reduction at Follow-up Visit by Phenotyping}

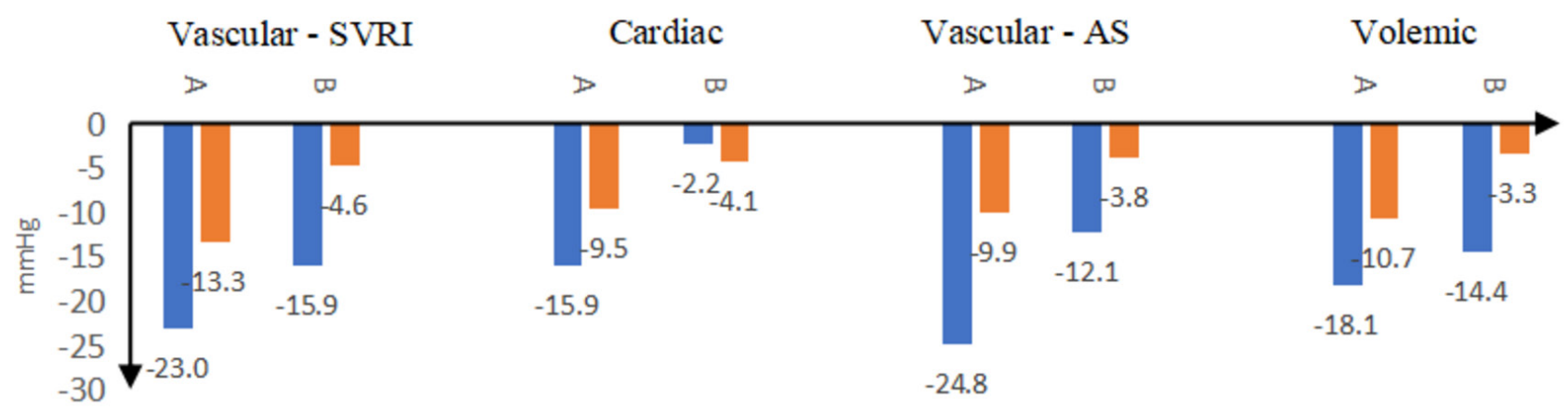

Figure 4 BP reduction by patients with different haemodynamic phenotypes. A: Haemodynamic group, B: standard care group. AS, arterial stiffness index; BP, blood pressure; DBP, diastolic BP; ICG, impedance cardiography; SBP, systolic BP; SVRI, systemic vascular resistance index. 

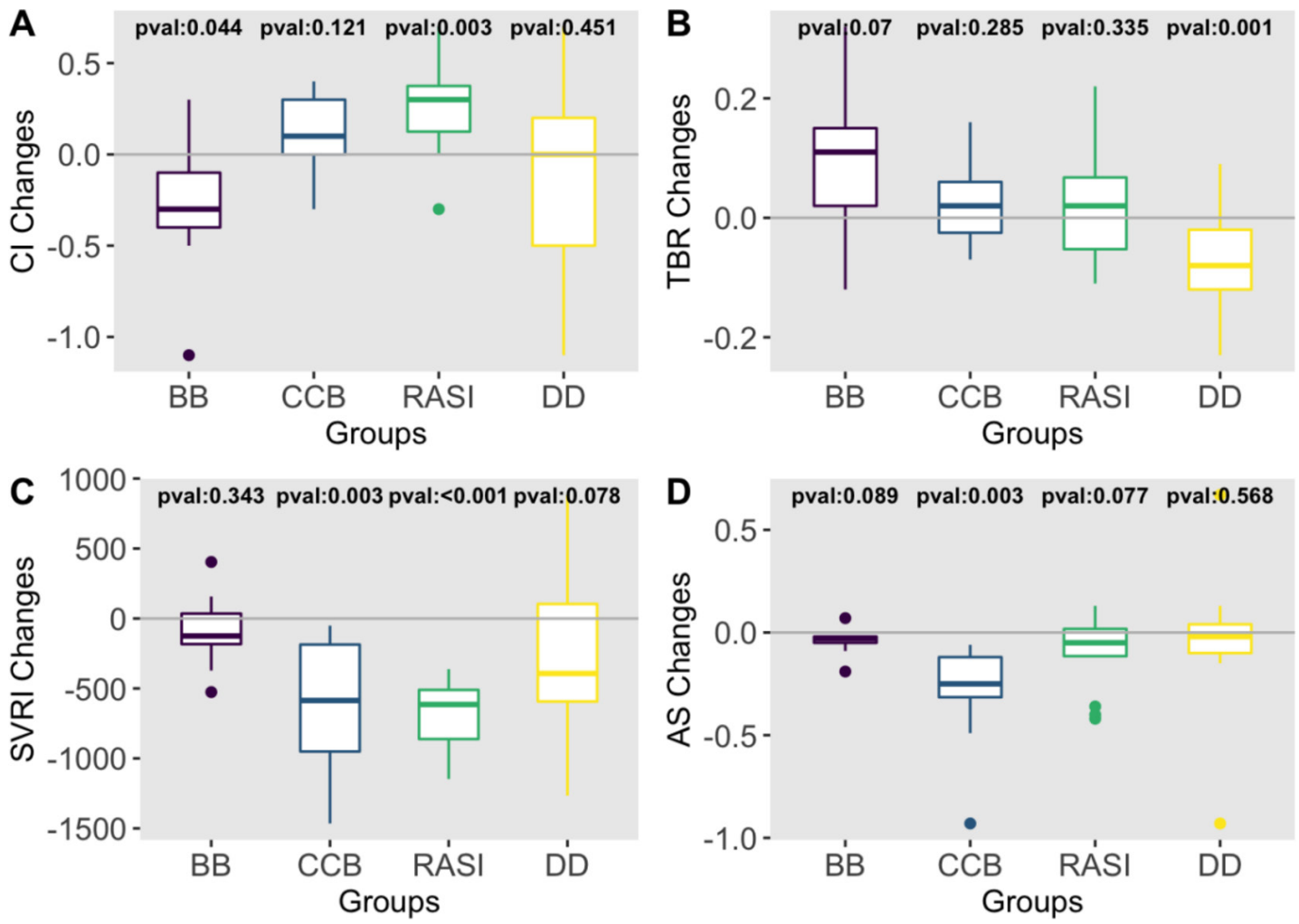

Figure 5 Impact of different antihypertensive agents on haemodynamic parameters. AS, arterial stiffness index; BB, betablockers; CB, calcium channel blockers; CI, cardiac index; DD, diuretics; RASI, renin-angiotensin system inhibitors; TBR, thoracic blood saturation ratio.

\section{Correlation between antihypertensive agents and changes in haemodynamic parameters}

In haemodynamic group, CI was statistically significantly reduced from baseline to follow-up visit in patients treated with beta-blockers $(\mathrm{p}=0.044$, figure 5$)$. TBR was statistically significantly reduced in patients treated with thiazide or thiazide-like diuretics $(\mathrm{p}=0.001)$. Both AS and SVRI were statistically significantly reduced in patients treated with calcium channel blockers $(p=0.003)$, and SVRI was statistically significantly reduced in patients treated with renin-angiotensin system inhibitors $(\mathrm{p}<0.001)$.

\section{DISCUSSION}

In a trial of stable hypertensive patients routinely seen in clinical practice in China, we showed that an ICGguided treatment strategy was more effective in reducing BP than standard therapy. These results were consistent across subgroups based on age, sex, BMI, baseline BP and haemodynamic phenotype. Our findings suggest that antihypertensive therapy tailored to each patient's haemodynamic abnormality could lead to more effective antihypertensive regimens and lay the groundwork for a more definitive trial. $^{892122}$

Of note, the reductions in $\mathrm{BP}$ in both groups were large, with an almost $20 \mathrm{~mm} \mathrm{Hg}$ decrease in SBP in the ICG-guided intervention group. The magnitude of the BP reduction in our study is largely consistent with previous studies conducted in the USA. Smith et al conducted a randomised controlled trial of 164 uncontrolled hypertensive patients on $1-3$ medications. ${ }^{8}$ After 3 months of treatment, patients in the ICG-guided group had an average SBP reduction of $19 \mathrm{~mm} \mathrm{Hg}$ compared with $12 \mathrm{~mm} \mathrm{Hg}$ in the standard care group. Taler et al randomised 104 patients with hypertension uncontrolled on two or more drugs to a 3-month trial of ICG-guided therapy or standard therapy directed by a hypertension specialist. ${ }^{9}$ In this study, the mean BP reduced from $169 / 87 \mathrm{~mm} \mathrm{Hg}$ to $139 / 72 \mathrm{~mm} \mathrm{Hg}$ in the ICG-guided group versus from $173 / 91 \mathrm{~mm} \mathrm{Hg}$ to $147 / 79 \mathrm{~mm} \mathrm{Hg}$ in the control group. We further extended previous studies by using a pragmatic design to test the ICG-guided intervention in real-life clinical practice and conducting the study in a low-income and middle-income country. We also provided a clinical decision support tool in addition to the ICG report to facilitate the antihypertensive treatment selection, producing a magnitude of BP improvement in routine clinical practice similar as that in the clinical trials.

There are several potential explanations for the findings in this study. First, the presumed mechanism for improved BP control with ICG-guided intervention is primarily due to personalised antihypertensive drug selection targeted at the haemodynamic cause of elevated BP. High BP results from one or more haemodynamic abnormality, including elevated CO, SVR and blood volume. ${ }^{23}$ Different antihypertensive agents act on different mechanisms to reduce $\mathrm{BP}$ by reducing $\mathrm{CO}$ or SVR. For example, beta-blockers block the effects of 
the hormone epinephrine and make the heart to beat more slowly and with less force, which then reduce $\mathrm{CO}$ and lower BP. Angiotensin-converting enzyme inhibitors (ACEIs)/ (angiotensin receptor blockers interfere with the body's renin-angiotensin-aldosterone system that leads to increased sodium and urine excreted, reduced resistance in blood vessels and increased venous capacity, which then reduce SVR and lower BP. Our ICG-guided intervention provides data on the underlying cause of elevated BP and uses clinical decision support to guide clinicians in selecting antihypertensive therapies targeted at the haemodynamic abnormality associated with the elevated BP, thereby maximising the BP lowering response for the given therapeutic selection.

Second, the larger reduction in BP in the intervention group may have been, in part, a reflection of improvement in therapeutic inertia. Therapeutic inertia, which refers to the failure of clinicians to initiate or intensify treatments when the BP is not at goal, has been showed as a most common cause of uncontrolled BP in actively treated patients. ${ }^{24} 25$ Providing clinician access to ICG findings of patients' haemodynamic profiles and clinical decision support tool for treatment selection may reduce therapeutic inertia in the intervention group.

Finally, the improvement of BP may, at least in part, be associated with improved communications and shared decision-making between the physician and the patient. The ICG report has served as a tool for physicians to communicate with and educate patients on the underlying haemodynamic abnormalities associated with their high BP and rationale for antihypertensive therapy selection in the intervention group. Previous studies have reported that patient-physician communication is an integral part of clinical practice and patients who understand explanations from their physicians are more likely to acknowledge health problems, modify behaviour and adhere to medications accordingly. ${ }^{26-28}$

Our findings have important clinical implications. Current diagnosis and management of hypertension are primary based on degree of BP elevation alone, with little attention paid to the underlying haemodynamic profile. Our study provides evidence for better identification of responders to a particular treatment regimen by profiling patients based on their haemodynamic profile using a simple, non-invasive test. As clinical care is moving towards precision medicine, our findings identify the needs of more refined haemodynamic measurement to facilitate personalised treatment in patients with hypertension. Additionally, the use of ICG-guided treatment strategy to achieve greater BP control offers a potential for better short-term use of healthcare resources. This is particularly relevant in low-income and middle-income counties where resources to improve hypertension control are limited and need to be more efficiently used. Given hypertension affects over one billion adults ( $30 \%$ of the global adult population) in the world ${ }^{29}$ such an approach has a large potential benefit in improving hypertension control and subsequently reducing a large number of cardiovascular events.
Several limitations should be considered in the interpretation of this study. First, this is a study with limited number of participants and relatively short follow-up. We did not collect long-term follow-up data, which could have been useful to assess the long-term effect of ICGguided treatment strategies in improving BP control. Second, our findings also warrant further study in other populations, as our study was conducted among a mostly urban, working class Chinese population and thus the results may not be generalisable to other populations. Third, we did not assess medication compliance among hypertensive patients, which may affect BP values of patients in the two arms. However, we used a pragmatic design to evaluate the effectiveness of interventions and we expect the medication compliance would be analogous to the scenarios in real-life routine clinical practice. Mediation refill rates were similar in the two arms as all patients fulfilled their prescriptions at the hospital pharmacy on the same day of the clinical encounters. Finally, we did not measure patients' behaviour change at home and patients may make lifestyle adjustment, such as reducing salt intake. However, this should be equally possible in both intervention and control groups given patients blinded in the trial.

In conclusion, a treatment strategy guided by haemodynamic measurements reduced BP more effectively than standard care in this trial in China. These findings justify further large-scale studies to provide more definitive evidence.

\section{Author affiliations}

${ }^{1}$ Department of Cardiology and Hypertension, Peking University People's Hospital, Beijing, China

${ }^{2}$ Section of Cardiovascular Medicine, Department of Internal Medicine, Yale School of Medicine, New Haven, Connecticut, USA

${ }^{3}$ Center for Outcomes Research and Evaluation, Yale-New Haven Hospital, New Haven, Connecticut, USA

${ }^{4}$ SJTU-Yale Joint Center for Biostatistics, Shanghai Jiao Tong University, Shanghai, China

${ }^{5}$ Shanghai Engineering Research Center for Big Data in Pediatric Precision Medicine, Shanghai, China

${ }^{6}$ Department of Research \& Development, Beijing Li-Heng Medical Technologies, Ltd, Beijing, China

${ }^{7}$ Department of Cardiology, Beijing Tiantan Puhua Hospital, Beijing, China ${ }^{8}$ Department of Health Policy and Management, Yale School of Medicine, New Haven, Connecticut, USA

Acknowledgements We thank all the subjects for their participation.

Contributors LW and YL contributed equally to the study. NS was responsible for study concept and design. LW and NS were responsible for the acquisition of data and its analysis. All Authors contributed to the interpretation of the data. YL drafted the manuscript. All authors performed critical revision of the manuscript for important intellectual content and approved the final manuscript. NS is the guarantor.

Funding This study was funded by an internal research grant from the Peking University People's Hospital. The funder had no relationship with the study planning, design and implementation. Dr Ningling Sun and Dr Luyan Wang had access to all the study data and Dr Wang was responsible for the data analysis.

Competing interests In the past three years, HK received expenses and/or personal fees from UnitedHealth, IBM Watson Health, Element Science, Aetna, Facebook, the Siegfried and Jensen Law Firm, Arnold and Porter Law Firm, Martin/ Baughman Law Firm, F-Prime, and the National Center for Cardiovascular Diseases 
in Beijing. He is an owner of Refactor Health and HugoHealth and had grants and/ or contracts from the Centers for Medicare \& Medicaid Services, Medtronic, the US Food and Drug Administration, Johnson \& Johnson and the Shenzhen Center for Health Information. YL is supported by the National Heart, Lung, and Blood Institute (K12HL138037) and the Yale Center for Implementation Science. She was a recipient of a research agreement, through Yale University, from the Shenzhen Center for Health Information for work to advance intelligent disease prevention and health promotion. ZJM is affiliated with Beijing Li-Heng Medical Technologies, Beijing, China.

Patient consent for publication Not required.

Ethics approval The study was reviewed and approved by the Peking University Institutional Review Board (reference ID: EC20190701). All hypertensive outpatients provided written informed consent and had study procedures consistent with the protocol.

Provenance and peer review Not commissioned; externally peer reviewed.

Data availability statement Data are available upon reasonable request. Technical appendix, statistical code and dataset are available upon request from the corresponding author.

Open access This is an open access article distributed in accordance with the Creative Commons Attribution 4.0 Unported (CC BY 4.0) license, which permits others to copy, redistribute, remix, transform and build upon this work for any purpose, provided the original work is properly cited, a link to the licence is given, and indication of whether changes were made. See: https://creativecommons.org/ licenses/by/4.0/.

Correction notice This article has been corrected since it was first published. The order of authors has been amended so that Dr. Luyan Wang is listed first and Dr. Yuan Lu is listed second.

ORCID iD

Yuan Lu http://orcid.org/0000-0001-5264-2169

\section{REFERENCES}

1 Oparil S, Zaman MA, Calhoun DA. Pathogenesis of hypertension. Ann Intern Med 2003;139:761-76.

2 Mahajan S, Gu J, Lu Y, et al. Hemodynamic phenotypes of hypertension based on cardiac output and systemic vascular resistance. Am J Med 2020;133:e127-39.

3 Mahajan S, Gu J, Caraballo C, et al. Relationship of age with the hemodynamic parameters in individuals with elevated blood pressure. J Am Geriatr Soc 2020;68:1520-8.

4 Drazner MH, Thompson B, Rosenberg PB, et al. Comparison of impedance cardiography with invasive hemodynamic measurements in patients with heart failure secondary to ischemic or nonischemic cardiomyopathy. Am J Cardiol 2002;89:993-5.

5 Sageman WS, Riffenburgh RH, Spiess BD. Equivalence of bioimpedance and Thermodilution in measuring cardiac index after cardiac surgery. J Cardiothorac Vasc Anesth 2002;16:8-14.

6 Ferrario CM, Smith RD, CONTROL Trial Investigators. Costeffectiveness of impedance cardiography testing in uncontrolled hypertension. Am Heart Hosp J 2006;4:279-89.

7 Ferrario CM, Flack JM, Strobeck JE, et al. Individualizing hypertension treatment with impedance cardiography: a metaanalysis of published trials. Ther Adv Cardiovasc Dis 2010;4:5-16.

8 Smith RD, Levy P, Ferrario CM, et al. Value of noninvasive hemodynamics to achieve blood pressure control in hypertensive subjects. Hypertension 2006;47:771-7.
9 Taler SJ, Textor SC, Augustine JE. Resistant hypertension: comparing hemodynamic management to specialist care. Hypertension 2002;39:982-8.

10 Bernstein DP. A new stroke volume equation for thoracic electrical bioimpedance: theory and rationale. Crit Care Med 1986;14:904-9.

11 Sherwood A, Allen MT, Fahrenberg J, et al. Methodological guidelines for impedance cardiography. Psychophysiology 1990;27:1-23.

12 Yancy C, Abraham WT. Noninvasive hemodynamic monitoring in heart failure: utilization of impedance cardiography. Congest Heart Fail 2003;9:241-50.

$13 \mathrm{Ma} \mathrm{L}$. Development and application of the latest model of the cardiac hemodynamics monitoring system. International $J$ Cardiovasc Med 2009;10:11.

14 X-g A, Zhao Y, Gao J. Clinic evaluation of noninvasive hemodynamic monitoring in patients undergoing coronary artery surgery. Chin $J$ Cardiovasc Rev 2008;2:010.

15 Hong H, Jin X-J, Pan C-Z, et al. [Analysis of the correlation between non-invasive hemodynamic monitor and cardiac echocardiography on the evaluation of cardiac function]. Zhongguo Yi Liao Qi Xie Za Zhi 2009;33:328-31.

16 Chen W, Huang D, Zeng J. Application of noninvasive cardiac hemodynamic monitor for ICU critically ill patients. Hainan Medical Journal 2018;29:15.

17 Liu L-S, Wu Z, Wang J, et al. 2018 Chinese guidelines for prevention and treatment of Hypertension-A report of the revision Committee of Chinese guidelines for prevention and treatment of hypertension. $J$ Geriatr Cardiol 2019;16:182-245.

18 Wannenburg T, Little WC. Regulation of cardiac output. In: Jeremias A, Brown DL, eds. Cardiac intensive care. 2nd edn. Philadelphia: W.B. Saunders, 2010: 61-8.

19 Gidwani UK, Mohanty B, Chatterjee K. The pulmonary artery catheter: a critical reappraisal. Cardiol Clin 2013;31:545-65.

20 Moher D, Schulz KF, Altman D, et al. The CONSORT statement: revised recommendations for improving the quality of reports of parallel-group randomized trials. JAMA 2001;285:1987-91.

21 Ventura HO, Taler SJ, Strobeck JE. Hypertension as a hemodynamic disease: the role of impedance cardiography in diagnostic, prognostic, and therapeutic decision making. Am J Hypertens 2005;18:26-43.

22 Sanford T, Treister N, Peters C. Use of noninvasive hemodynamics in hypertension management. Am J Hypertens 2005;18:87-91.

23 Mayet J, Hughes A. Cardiac and vascular pathophysiology in hypertension. Heart 2003;89:1104-9.

24 O'Connor PJ. Overcome clinical inertia to control systolic blood pressure. Arch Intern Med 2003;163:2677-8.

25 Okonofua EC, Simpson KN, Jesri A, et al. Therapeutic inertia is an impediment to achieving the healthy people 2010 blood pressure control goals. Hypertension 2006;47:345-51.

26 Travaline JM, Ruchinskas R, D'Alonzo GE. Patient-Physician communication: why and how. J Am Osteopath Assoc 2005;105:13-18.

27 Bull SA, Hu XH, Hunkeler EM, et al. Discontinuation of use and switching of antidepressants: influence of patient-physician communication. JAMA 2002;288:1403-9.

28 Ciechanowski PS, Katon WJ, Russo JE, et al. The patient-provider relationship: attachment theory and adherence to treatment in diabetes. Am J Psychiatry 2001:158:29-35.

29 NCD Risk Factor Collaboration (NCD-RisC). Worldwide trends in blood pressure from 1975 to 2015: a pooled analysis of 1479 population-based measurement studies with $19 \cdot 1$ million participants. Lancet 2017;389:37-55. 
Correction: Effectiveness of an impedance cardiography guided treatment strategy to improve blood pressure control in a real-world setting: results from a pragmatic clinical trial

Lu Y, Wang L, Wang $\mathrm{H}$, et al. Effectiveness of an impedance cardiography guided treatment strategy to improve blood pressure control in a real-world setting: results from a pragmatic clinical trial. Open Heart 2021;8:e001719. doi:10.1136/openhrt2021-001719

This article has been corrected since it was first published. The order of authors has been amended so that Dr. Luyan Wang is listed first and Dr. Yuan Lu is listed second.

Open access This is an open access article distributed in accordance with the Creative Commons Attribution 4.0 Unported (CC BY 4.0) license, which permits others to copy, redistribute, remix, transform and build upon this work for any purpose, provided the original work is properly cited, a link to the licence is given, and indication of whether changes were made. See: https:// creativecommons.org/licenses/by/4.0/.

C Author(s) (or their employer(s)) 2021. Re-use permitted under CC BY. Published by BMJ.

Open Heart 2021;8:e001719corr1. doi:10.1136/openhrt-2021-001719corr1

(A) Check for updates 
Correction: Effectiveness of an impedance cardiography guided treatment strategy to improve blood pressure control in a real-world setting: results from a pragmatic clinical trial

Lu Y, Wang L, Wang $\mathrm{H}$, et al. Effectiveness of an impedance cardiography guided treatment strategy to improve blood pressure control in a real-world setting: results from a pragmatic clinical trial. Open Heart 2021;8:e001719. doi:10.1136/openhrt2021-001719

This article has been corrected since it was first published. The order of authors has been amended so that Dr. Luyan Wang is listed first and Dr. Yuan Lu is listed second.

Open access This is an open access article distributed in accordance with the Creative Commons Attribution 4.0 Unported (CC BY 4.0) license, which permits others to copy, redistribute, remix, transform and build upon this work for any purpose, provided the original work is properly cited, a link to the licence is given, and indication of whether changes were made. See: https:// creativecommons.org/licenses/by/4.0/.

C Author(s) (or their employer(s)) 2021. Re-use permitted under CC BY. Published by BMJ.

Open Heart 2021;8:e001719corr2. doi:10.1136/openhrt-2021-001719corr2

A) Check for updates 